(153)

\title{
Fabrication of Antimicrobial Material for Food Packaging Applications
}

\author{
Egodage D.P., Jayalath H.T.S., Samarasekara A.M.P.B." and Amarasinghe D.A.S. \\ Department of Materials Science and Engineering, University of Moratuwa, Sri Lanka \\ *banduamp@yahoo.com
}

\begin{abstract}
Nowadays, polystyrene is the widely used material in disposable food packaging applications. One of the main disadvantages of polystyrene as a food packaging material is the microbial attack during the food storage. Objective of this research is to fabricate a nano silver based antimicrobial coating on polystyrene based packaging material to extent the self-life. In this research nano silver solution was prepared using polyvinyl alcohol and silver nitrate. Nano silver formation was confirmed by analyzing UV-Vis spectrum. Citric acid was used as the crosslinking agent of polyvinyl alcohol (PVA). UV assisted treatment was used to modify the internal surface of the polystyrene containers to improve the wettability. Fourier transforms infrared spectroscopy - Attenuated total reflection method was used to identify the surface modifications after the UV treatment. Fourier transform infrared spectroscopy test results showed the significant changes occurred on internal surface of Polystyrene samples due to UV treatment. The prepared Ag/PVA/Citric solution was coated on UV treated polystyrene container and kept under the heat to crosslink with the surface. Antimicrobial activity of prepared nano silver coated polystyrene based food packaging was tested against E.coli bacteria. There was a clear inhibition zone in nano silver coated Polystyrene sample under $E$. coli growth. It indicated that nano silver coated polystyrene containers showed significant antimicrobial activity. There will be various human health, safety, minimum waste generation and economic benefits from these developed nano silver coated polystyrene food packaging products. These products can be used to control the waste generation in near future.
\end{abstract}

Keywords: Food packaging, Nano silver, Antimicrobial, Polystyrene

Proceedings of the International Forestry and Environment Symposium 2016, Department of Forestry and Environmental Science, University of Sri Jayewardenepura, Sri Lanka. 